\title{
A Generalized Skew Information and Uncertainty Relation
}

\author{
Kenjiro Yanagi, Member, IEEE, Shigeru Furuichi, Member, IEEE,
} and Ken Kuriyama

\begin{abstract}
A generalized skew information is defined and a generalized uncertainty relation is established with the help of a trace inequality which was recently proven by Fujii. In addition, we prove the trace inequality conjectured by Luo and Zhang. Finally, we point out that Theorem 1 in S. Luo and Q. Zhang, IEEE Trans. Inf. Theory, vol. 50, pp. 1778-1782, no. 8, Aug. 2004 is incorrect in general, by giving a simple counter-example.
\end{abstract}

Index Terms-Skew information, trace inequalities and uncertainty relation.

\section{INTRODUCTION}

As one of the mathematical studies on entropy, the skew entropy [14], [15] and the problem of its concavity are famous. The concavity problem for the skew entropy generalized by Dyson, was solved by Lieb in [9]. It is also known that the skew entropy represents the degree of noncommutativity between a certain quantum state represented by the density matrix $\rho$ (which is a positive semidefinite matrix with unit trace) and an observable represented by the selfadjoint matrix $X$. Quite recently, S. Luo and Q. Zhang studied the relation between skew information (which is equal to the opposite signed skew entropy) and the uncertainty relation in [10]. Inspired by their interesting work, we define a generalized skew information and then study the relationship between it and the uncertainty relation. In addition, we prove the trace inequality conjectured in [11].

\section{PRELIMINARIES}

Let $f$ and $g$ be functions on the domain $D \subset \boldsymbol{R} .(f, g)$ is called a monotonic pair if $(f(a)-f(b))(g(a)-g(b)) \geq 0$ for all $a, b \in D .(f, g)$ is also called an antimonotonic pair if $(f(a)-f(b))(g(a)-g(b)) \leq 0$ for all $a, b \in D$.

In what follows we consider selfadjoint matrices whose spectra are included in $D$ so that functional calculus makes sense.

Lemma II.1 ([1], [2]): For any selfadjoint matrices $A$ and $X$, we have the following trace inequalities.

1) If $(f, g)$ is a monotonic pair, then

$$
\operatorname{Tr}(f(A) X g(A) X) \leq \operatorname{Tr}\left(f(A) g(A) X^{2}\right)
$$

2) If $(f, g)$ is an antimonotonic pair, then

$$
\operatorname{Tr}(f(A) X g(A) X) \geq \operatorname{Tr}\left(f(A) g(A) X^{2}\right)
$$

From this lemma, we can obtain the following lemma.

Manuscript received January 26, 2005; revised August 10, 2005.

K. Yanagi and K. Kuriyama are with the Department of Applied Science, Yamaguchi University, Yamaguchi 755-8611, Japan (e-mail: yanagi@yamaguchiu.ac.jp; kuriyama@yamaguchi-u.ac.jp).

S. Furuichi is with the Department of Electronics and Computer Science, Tokyo University of Science, Yamaguchi, SanyoOnoda City 756-0884, Japan (e-mail: furuichi@ed.yama.tus.ac.jp).

Communicated by E. Knill, Associate Editor for Quantum Information Theory.

Digital Object Identifier 10.1109/TIT.2005.858971 
Lemma II.2: For any selfadjoint matrices $A$ and $B$, and any matrix $X$, we have the following trace inequalities.

1) If $(f, g)$ is a monotonic pair, then

$$
\begin{aligned}
\operatorname{Tr}\left(f(A) X^{*} g(B) X+f(B) X g(A) X^{*}\right) \\
\leq \\
\leq \operatorname{Tr}\left(f(A) g(A) X^{*} X+f(B) g(B) X X^{*}\right) .
\end{aligned}
$$

2) If $(f, g)$ is an antimonotonic pair, then

$$
\begin{aligned}
\operatorname{Tr}\left(f(A) X^{*} g(B) X+f(B) X g(A) X^{*}\right) \\
\geq \operatorname{Tr}\left(f(A) g(A) X^{*} X+f(B) g(B) X X^{*}\right) .
\end{aligned}
$$

Proof: Define on $\mathcal{H} \oplus \mathcal{H}$

$$
\hat{A}=\left(\begin{array}{cc}
A & 0 \\
0 & B
\end{array}\right), \quad \hat{X}=\left(\begin{array}{cc}
0 & X^{*} \\
X & 0
\end{array}\right)
$$

where $A, B$ and $X$ act on a finite-dimensional Hilbert space $\mathcal{H}$. Then $\hat{A}$ and $\hat{X}$ are selfadjoint. Therefore, one may apply Lemma II.1 to get

$$
\begin{aligned}
\operatorname{Tr} & \left.f(A) X^{*} g(B) X+f(B) X g(A) X^{*}\right) \\
= & \operatorname{Tr}\left(\left(\begin{array}{cc}
f(A) & 0 \\
0 & f(B)
\end{array}\right)\left(\begin{array}{cc}
0 & X^{*} \\
X & 0
\end{array}\right)\right. \\
& \left.\quad\left(\begin{array}{cc}
g(A) & 0 \\
0 & g(B)
\end{array}\right)\left(\begin{array}{cc}
0 & X^{*} \\
X & 0
\end{array}\right)\right) \\
= & \operatorname{Tr}(f(\hat{A}) \hat{X} g(\hat{A}) \hat{X}) \\
\leq & \operatorname{Tr}\left(f(\hat{A}) g(\hat{A}) \hat{X}^{2}\right) \\
= & \operatorname{Tr}\left(\left(\begin{array}{cc}
f(A) & 0 \\
0 & f(B)
\end{array}\right)\left(\begin{array}{cc}
g(A) & 0 \\
0 & g(B)
\end{array}\right)\right. \\
& \left.\quad\left(\begin{array}{cc}
0 & X^{*} \\
X & 0
\end{array}\right)\left(\begin{array}{cc}
0 & X^{*} \\
X & 0
\end{array}\right)\right) \\
= & \operatorname{Tr}\left(f(A) g(A) X^{*} X+f(B) g(B) X X^{*}\right)
\end{aligned}
$$

which is (1). Inequality (2) is proven in a similar way.

\section{GENERALIZED UNCERTAINTY RELATION}

For a density matrix (quantum state) $\rho$ and arbitrary matrices $X$ and $Y$ acting on $\mathcal{H}$, we denote $\tilde{X} \equiv X-\operatorname{Tr}(\rho X) I$ and $\tilde{Y} \equiv Y-\operatorname{Tr}(\rho Y) I$, where $I$ represents the identity matrix. Then we define the covariance by $\operatorname{Cov}_{\rho}(X, Y)=\operatorname{Tr}(\rho \tilde{X} \tilde{Y})$. Each variance is defined by $V_{\rho}(X) \equiv$ $\operatorname{Cov}_{\rho}(X, X)$ and $V_{\rho}(Y) \equiv \operatorname{Cov}_{\rho}(Y, Y)$.

The famous Heisenberg's uncertainty relation [6], [12] can be easily proven by the application of the Schwarz inequality and it was generalized by Schrödinger as follows:

Propostion III.1 (Schrödinger [13]): For any density matrix $\rho$ and any two selfadjoint matrices $A$ and $B$, we have the uncertainty relation

$$
V_{\rho}(A) V_{\rho}(B)-\left|\operatorname{Re}\left(\operatorname{Cov}_{\rho}(A, B)\right)\right|^{2} \geq \frac{1}{4}|\operatorname{Tr}(\rho[A, B])|^{2}
$$

where $[X, Y] \equiv X Y-Y X$.

Definition III.2: For arbitrary matrices $X$ and $Y$, we define

$$
I_{p}(\rho ; X, Y) \equiv \operatorname{Tr}(\rho X Y)-\operatorname{Tr}\left(\rho^{\frac{1}{p}} X \rho^{\frac{1}{p^{*}}} Y\right)
$$

where $p \in[1,+\infty]$ and with $p^{*}$ such that $\frac{1}{p}+\frac{1}{p^{*}}=1$. If $A$ is selfadjoint, the Wigner-Yanase-Dyson information is defined by

$$
\begin{aligned}
I_{p}(\rho ; A) & \equiv I_{p}(\rho ; A, A)=\operatorname{Tr}\left(\rho A^{2}\right)-\operatorname{Tr}\left(\rho^{\frac{1}{p}} A \rho^{\frac{1}{p^{*}}} A\right) \\
& =-\frac{1}{2} \operatorname{Tr}\left(\left[\rho^{\frac{1}{p}}, A\right]\left[\rho \frac{1}{p^{*}}, A\right]\right) .
\end{aligned}
$$

We use the parameters $p$ and $p^{*}$, since many papers [3]-[5], [7] in this field use such notations. The Wigner-Yanase skew information is

$$
\begin{aligned}
I(\rho ; A) & \equiv I_{2}(\rho ; A)=\operatorname{Tr}\left(\rho A^{2}\right)-\operatorname{Tr}\left(\rho^{\frac{1}{2}} A \rho^{\frac{1}{2}} A\right) \\
& =-\frac{1}{2} \operatorname{Tr}\left(\left[\rho^{\frac{1}{2}}, A\right]^{2}\right) .
\end{aligned}
$$

An interpretation of skew information as a measure of quantum uncertainty is given in [10]. They claimed the following uncertainty relation

$$
I(\rho, A) I(\rho, B)-\left|\operatorname{Re}\left(\operatorname{Corr}_{\rho}(A, B)\right)\right|^{2} \geq \frac{1}{4}|\operatorname{Tr}(\rho[A, B])|^{2}
$$

for two selfadjoint matrices $A$ and $B$, and density matrix $\rho$, where their correlation measure was defined by

$$
\operatorname{Corr}_{\rho}(A, B) \equiv \operatorname{Tr}(\rho A B)-\operatorname{Tr}\left(\rho^{1 / 2} A \rho^{1 / 2} B\right)
$$

However, we show (2) does not hold in general. We give a counterexample for (2) in Section IV.

We define the generalized skew correlation and the generalized skew information as follows.

Definition III.3: For arbitrary $X$ and $Y, p \in[1,+\infty]$ with $p^{*}$ such that $\frac{1}{p}+\frac{1}{p^{*}}=1$ and $\varepsilon \geq 0$, set

$\phi_{p, \varepsilon}(\rho ; X, Y) \equiv \varepsilon \operatorname{Cov}_{\rho}\left(X^{*}, Y\right)+\frac{1}{2} I_{p}\left(\rho ; \tilde{X}^{*}, \tilde{Y}\right)+\frac{1}{2} I_{p}\left(\rho ; \tilde{Y}, \tilde{X}^{*}\right)$.

If $A$ and $B$ are selfadjoint, the generalized skew correlation is defined by

$$
\operatorname{Corr}_{p, \varepsilon}(\rho ; A, B) \equiv \phi_{p, \varepsilon}(\rho ; A, B) \text {. }
$$

The generalized skew information is defined by

$$
I_{p, \varepsilon}(\rho ; A) \equiv \operatorname{Corr}_{p, \varepsilon}(\rho ; A, A)=\varepsilon V_{\rho}(A)+I_{p}(\rho ; \tilde{A})
$$

so that

$$
I_{p, 0}(\rho ; A)=I_{p}(\rho ; \tilde{A})=V_{\rho}(A)-\operatorname{Tr}\left(\rho^{\frac{1}{p}} \tilde{A} \rho^{\frac{1}{p^{*}} \tilde{A}}\right) .
$$

Then we have the following theorem.

Theorem III.4: For any two selfadjoint matrices $A$ and $B$, any density matrix $\rho$, any $p \in[1,+\infty]$ with $p^{*}$ such that $\frac{1}{p}+\frac{1}{p^{*}}=1$ and $\varepsilon \geq 0$, we have a generalized uncertainty relation

$I_{p, \varepsilon}(\rho ; A) I_{p, \varepsilon}(\rho ; B)-\left|\operatorname{Re}\left(\operatorname{Corr}_{p, \varepsilon}(\rho ; A, B)\right)\right|^{2} \geq \frac{\varepsilon^{2}}{4}|\operatorname{Tr}(\rho[A, B])|^{2}$. 
Proof: By Lemma II.2, $\phi_{p, \varepsilon}(\rho ; X, X) \geq 0$. Furthermore it is clear that $\phi_{p, \varepsilon}(\rho ; X, Y)$ is sesquilinear and Hermitian. Then we have

$$
\mid \phi_{p, \varepsilon}(\rho ; X, Y)^{2} \leq \phi_{p, \varepsilon}(\rho ; X, X) \phi_{p, \varepsilon}(\rho ; Y, Y)
$$

by the Schwarz inequality. It follows that

$$
\left|\operatorname{Corr}_{p, \varepsilon}(\rho ; A, B)\right|^{2} \leq \operatorname{Corr}_{p, \varepsilon}(\rho ; A, A) \operatorname{Corr}_{p, \varepsilon}(\rho ; B, B)
$$

for any two selfadjoint matrices $A$ and $B$. Then

$$
\left|\operatorname{Corr}_{p, \varepsilon}(\rho ; A, B)\right|^{2} \leq I_{p, \varepsilon}(\rho ; A) I_{p, \varepsilon}(\rho ; B) .
$$

Simple calculations imply

$\operatorname{Corr}_{p, \varepsilon}(\rho ; A, B)-\operatorname{Corr}_{p, \varepsilon}(\rho ; B, A)=\varepsilon \operatorname{Tr}(\rho[\tilde{A}, \tilde{B}])=\varepsilon \operatorname{Tr}(\rho[A, B])$

$\operatorname{Corr}_{p, \varepsilon}(\rho ; A, B)+\operatorname{Corr}_{p, \varepsilon}(\rho ; B, A)=2 \operatorname{Re}\left(\operatorname{Corr}_{p, \varepsilon}(\rho ; A, B)\right)$.

Summing both sides in the above two equalities, we have

$$
2 \operatorname{Corr}_{p, \varepsilon}(\rho ; A, B)=\varepsilon \operatorname{Tr}(\rho[A, B])+2 \operatorname{Re}\left(\operatorname{Corr}_{p, \varepsilon}(\rho ; A, B)\right)
$$

Since $[A, B]$ is skew-adjoint, $\operatorname{Tr}(\rho[A, B])$ is a purely imaginary number, we have

$\left|\operatorname{Corr}_{p, \varepsilon}(\rho ; A, B)\right|^{2}=\frac{\varepsilon^{2}}{4}|\operatorname{Tr}(\rho[A, B])|^{2}+\left|\operatorname{Re}\left(\operatorname{Corr}_{p, \varepsilon}(\rho ; A, B)\right)\right|^{2}$.

Thus the proof of the theorem is completed by the use of (3) and (7).

We are interested in the relationship between the left-hand sides in Proposition III.1 and Theorem III.4. The following proposition gives the relationship.

Proposition III.5: For any two selfadjoint matrices $A$ and $B$, any density matrix $\rho$, any $p \in[1,+\infty]$ with $p^{*}$ such that $\frac{1}{p}+\frac{1}{p^{*}}=1$ and $\varepsilon \geq 0$, we have

$$
\begin{aligned}
I_{p, \varepsilon}(\rho ; A) I_{p, \varepsilon}(\rho ; B)- & \left|\operatorname{Re}\left(\operatorname{Corr}_{p, \varepsilon}(\rho ; A, B)\right)\right|^{2} \\
& \geq \varepsilon^{2} V_{\rho}(A) V_{\rho}(B)-\varepsilon^{2}\left|\operatorname{Re}\left(\operatorname{Cov}_{\rho}(A, B)\right)\right|^{2} .
\end{aligned}
$$

Proof: From Proposition III.1, we have

$$
V_{\rho}(A) V_{\rho}(B) \geq\left|\operatorname{Re}\left(\operatorname{Cov}_{\rho}(A, B)\right)\right|^{2}
$$

that is,

$$
|\operatorname{Re}(\operatorname{Tr}(\rho \tilde{A} \tilde{B}))|^{2} \leq \operatorname{Tr}\left(\rho \tilde{A}^{2}\right) \operatorname{Tr}\left(\rho \tilde{B}^{2}\right) .
$$

By putting $\varepsilon=0$ in (3), we have

$$
\left|\operatorname{Corr}_{p, 0}(\rho ; A, B)\right|^{2} \leq I_{p, 0}(\rho ; A) I_{p, 0}(\rho ; B) .
$$

It follows from (4) and (5) that

$$
\operatorname{Corr}_{p, 0}(\rho ; A, B)=\operatorname{Re}\left(\operatorname{Corr}_{p, 0}(\rho ; A, B)\right) .
$$

Thus,

$$
\left|\operatorname{Re}\left(\operatorname{Corr}_{p, 0}(\rho ; A, B)\right)\right|^{2} \leq I_{p, 0}(\rho ; A) I_{p, 0}(\rho ; B) .
$$

Using (8), (9) and direct calculations, we get

$$
\begin{aligned}
\text { L.H.S. } & - \text { R.H.S. } \\
= & \varepsilon \operatorname{Tr}\left(\rho \tilde{A}^{2}\right) I_{p, 0}(\rho ; B)+\varepsilon \operatorname{Tr}\left(\rho \tilde{B}^{2}\right) I_{p, 0}(\rho ; A) \\
& -2 \varepsilon \operatorname{Re}(\operatorname{Tr}(\rho \tilde{A} \tilde{B})) \operatorname{Re}\left(\operatorname{Corr}_{p, 0}(\rho ; A, B)\right) \\
& +I_{p, 0}(\rho ; A) I_{p, 0}(\rho ; B)-\left\{\operatorname{Re}\left(\operatorname{Corr}_{p, 0}(\rho ; A, B)\right)\right\}^{2} \\
\geq & \varepsilon \operatorname{Tr}\left(\rho \tilde{A}^{2}\right) I_{p, 0}(\rho ; B)+\varepsilon \operatorname{Tr}\left(\rho \tilde{B}^{2}\right) I_{p, 0}(\rho ; A) \\
& -2 \varepsilon \operatorname{Re}(\operatorname{Tr}(\rho \tilde{A} \tilde{B})) \operatorname{Re}\left(\operatorname{Corr}_{p, 0}(\rho ; A, B)\right) \\
\geq & \varepsilon \operatorname{Tr}\left(\rho \tilde{A}^{2}\right) I_{p, 0}(\rho ; B)+\varepsilon \operatorname{Tr}\left(\rho \tilde{B}^{2}\right) I_{p, 0}(\rho ; A) \\
& -2 \varepsilon \sqrt{\operatorname{Tr}\left(\rho \tilde{A}^{2}\right) \operatorname{Tr}\left(\rho \tilde{B}^{2}\right)} \sqrt{I_{p, 0}(\rho ; A) I_{p, 0}(\rho ; B)} \\
= & \varepsilon\left\{\sqrt{\operatorname{Tr}\left(\rho \tilde{A}^{2}\right) I_{p, 0}(\rho ; B)}-\sqrt{\operatorname{Tr}\left(\rho \tilde{B}^{2}\right) I_{p, 0}(\rho ; A)}\right\}^{2} \\
\geq & 0 .
\end{aligned}
$$

Remark III.6: Theorem III.4 can be also proven by Proposition III.1 and Proposition III.5.

\section{AN INEQUALITY RELATED TO THE UNCERTAINTY RELATION}

The trace inequality

$$
\begin{aligned}
V_{\rho}(A) V_{\rho}(B)- & \left|\operatorname{Re}\left(\operatorname{Cov}_{\rho}(A, B)\right)\right|^{2} \\
& \geq I_{2,0}(\rho ; A) I_{2,0}(\rho ; B)-\left|\operatorname{Re}\left(\operatorname{Corr}_{2,0}(\rho ; A, B)\right)\right|^{2} .
\end{aligned}
$$

was conjectured in [11] and proven in [10]. As a generalization of [10, Theorem 2], we prove a one-parameter extention of the above inequality.

Proposition IV.1: For any two selfadjoint matrices $A$ and $B$, any density matrix $\rho$ and any $p \in[1,+\infty]$ with $p^{*}$ such that $\frac{1}{p}+\frac{1}{p^{*}}=1$, we have

$$
\begin{aligned}
& V_{\rho}(A) V_{\rho}(B)-\left|\operatorname{Re}\left(\operatorname{Cov}_{\rho}(A, B)\right)\right|^{2} \\
& \quad \geq I_{p, 0}(\rho ; A) I_{p, 0}(\rho ; B)-\left|\operatorname{Re}\left(\operatorname{Corr}_{p, 0}(\rho ; A, B)\right)\right|^{2} .
\end{aligned}
$$

Proof: Let $\left\{\varphi_{i}\right\}$ be a complete orthonormal basis composed by eigenvectors of $\rho$. Then we calculate

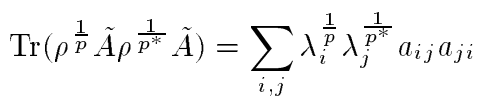

where $a_{i j} \equiv\left\langle\tilde{A} \varphi_{i} \mid \varphi_{j}\right\rangle$ and $a_{j i} \equiv \overline{a_{i j}}$. Thus, we get

$$
\begin{aligned}
& I_{p, 0}(\rho ; A)=V_{\rho}(A)-\sum_{i, j} \lambda_{i}^{\frac{1}{p}} \lambda_{j}^{\frac{1}{p^{*}}} a_{i j} a_{j i} \\
& I_{p, 0}(\rho ; B)=V_{\rho}(B)-\sum_{i, j} \lambda_{i}^{\frac{1}{p}} \lambda_{j}^{\frac{1}{p^{*}}} b_{i j} b_{j i}
\end{aligned}
$$


where $b_{i j} \equiv\left\langle\tilde{B} \varphi_{i} \mid \varphi_{j}\right\rangle$ and $b_{j i} \equiv \overline{b_{i j}}$. In a similar way, we obtain

$$
\begin{aligned}
& \operatorname{Re}\left(\operatorname{Corr}_{p, 0}(\rho ; A, B)\right)=\operatorname{Re}\left(\operatorname{Cov}_{\rho}(A, B)\right) \\
& -\frac{1}{2} \sum_{i, j} \lambda_{i}^{\frac{1}{p}} \lambda_{j}^{\frac{1}{p^{*}}} \operatorname{Re}\left(a_{i j} b_{j i}\right) \\
& -\frac{1}{2} \sum_{j, i} \lambda_{i}^{\frac{1}{p}} \lambda_{j}^{\frac{1}{p^{*}}} \operatorname{Re}\left(b_{i j} a_{j i}\right) .
\end{aligned}
$$

In order to prove the present proposition, we have only to show the inequality $\xi \geq \eta$, where

$$
\begin{aligned}
\xi \equiv & V_{\rho}(A) \sum_{i, j} \lambda_{i}^{\frac{1}{p}} \lambda_{j}^{\frac{1}{p^{*}}} b_{i j} b_{j i}+V_{\rho}(B) \sum_{i, j} \lambda_{i}^{\frac{1}{p}} \lambda_{j}^{\frac{1}{p^{*}}} a_{i j} a_{j i} \\
& -\left(\sum_{i, j} \lambda_{i}^{\frac{1}{p}} \lambda_{j}^{\frac{1}{p^{*}}} a_{i j} a_{j i}\right)\left(\sum_{i, j} \lambda_{i}^{\frac{1}{p}} \lambda_{j}^{\frac{1}{p^{*}}} b_{i j} b_{j i}\right), \\
\eta \equiv & \operatorname{Re}\left(\operatorname{Cov}_{\rho}(A, B)\right) \sum_{i, j} \lambda_{i}^{\frac{1}{p}} \lambda_{j}^{\frac{1}{p^{*}}} \operatorname{Re}\left(a_{i j} b_{j i}\right) \\
& +\operatorname{Re}\left(\operatorname{Cov}_{\rho}(A, B)\right) \sum_{i, j} \lambda_{i}^{\frac{1}{p}} \lambda_{j}^{\frac{1}{p^{*}}} \operatorname{Re}\left(b_{i j} a_{j i}\right) \\
& -\frac{1}{4}\left(\sum_{i, j} \lambda_{i}^{\frac{1}{p}} \lambda_{j}^{\frac{1}{p^{*}}} \operatorname{Re}\left(a_{i j} b_{j i}\right)+\sum_{i, j} \lambda_{i}^{\frac{1}{p}} \lambda_{j}^{\frac{1}{p^{*}}} \operatorname{Re}\left(b_{i j} a_{j i}\right)\right)^{2} .
\end{aligned}
$$

Since

$$
\begin{aligned}
& V_{\rho}(A)=\operatorname{Tr}\left(\rho \tilde{A}^{2}\right)=\frac{1}{2} \sum_{i, j}\left(\lambda_{i}+\lambda_{j}\right) a_{i j} a_{j i} \\
& V_{\rho}(B)=\operatorname{Tr}\left(\rho \tilde{B}^{2}\right)=\frac{1}{2} \sum_{i, j}\left(\lambda_{i}+\lambda_{j}\right) b_{i j} b_{j i}
\end{aligned}
$$

and

$$
\left(\lambda_{i}+\lambda_{j}\right) \lambda_{k}^{\frac{1}{p}} \lambda_{l}^{\frac{1}{p^{*}}}+\left(\lambda_{k}+\lambda_{l}\right) \lambda_{i}^{\frac{1}{p}} \lambda_{j}^{\frac{1}{p^{*}}}-2 \lambda_{i}^{\frac{1}{p}} \lambda_{j}^{\frac{1}{p^{*}}} \lambda_{k}^{\frac{1}{p}} \lambda_{l}^{\frac{1}{p^{*}}} \geq 0
$$

we calculate

$$
\begin{gathered}
\xi=\frac{1}{4} \sum_{i, j, k, l}\left\{\left(\lambda_{i}+\lambda_{j}\right) \lambda_{k}^{\frac{1}{p}} \lambda_{l}^{\frac{1}{p^{*}}}+\left(\lambda_{k}+\lambda_{l}\right) \lambda_{i}^{\frac{1}{p}} \lambda_{j}^{\frac{1}{p^{*}}}\right. \\
\left.-2 \lambda_{i}^{\frac{1}{p}} \lambda_{j}^{\frac{1}{p^{*}}} \lambda_{k}^{\frac{1}{p}} \lambda_{l}^{\frac{1}{p^{*}}}\right\}\left(a_{i j} a_{j i} b_{k l} b_{l k}+b_{i j} b_{j i} a_{k l} a_{l k}\right) \\
\geq \frac{1}{2} \sum_{i, j, k, l}\left\{\left(\lambda_{i}+\lambda_{j}\right) \lambda_{k}^{\frac{1}{p}} \lambda_{l}^{\frac{1}{p^{*}}}+\left(\lambda_{k}+\lambda_{l}\right) \lambda_{i}^{\frac{1}{p}} \lambda_{j}^{\frac{1}{p^{*}}}\right. \\
\left.-2 \lambda_{i}^{\frac{1}{p}} \lambda_{j}^{\frac{1}{p^{*}}} \lambda_{k}^{\frac{1}{p}} \lambda_{l}^{\frac{1}{p^{*}}}\right\}\left|a_{i j} b_{j i}\right|\left|a_{k l} b_{l k}\right| .
\end{gathered}
$$

Since $\operatorname{Re}\left(b_{k l} a_{l k}\right)=\operatorname{Re}\left(\overline{b_{l k}} \overline{a_{k l}}\right)=\operatorname{Re}\left(b_{l k} a_{k l}\right)=\operatorname{Re}\left(a_{k l} b_{l k}\right)$, $\operatorname{Re}\left(b_{i j} a_{j i}\right)=\operatorname{Re}\left(a_{i j} b_{j i}\right)$, we calculate

$$
\begin{array}{r}
\eta=\frac{1}{2} \sum_{i, j, k, l}\left\{\left(\lambda_{i}+\lambda_{j}\right) \lambda_{k}^{\frac{1}{p}} \lambda_{l}^{\frac{1}{p^{*}}}+\left(\lambda_{k}+\lambda_{l}\right) \lambda_{i}^{\frac{1}{p}} \lambda_{j}^{\frac{1}{p^{*}}}\right. \\
\left.-2 \lambda_{i}^{\frac{1}{p}} \lambda_{j}^{\frac{1}{p^{*}}} \lambda_{k}^{\frac{1}{p}} \lambda_{l}^{\frac{1}{p^{*}}}\right\} \operatorname{Re}\left(a_{i j} b_{j i}\right) \operatorname{Re}\left(a_{k l} b_{l k}\right) .
\end{array}
$$

Thus, we conclude $\xi \geq \eta$, since

$$
\left|a_{i j} b_{j i}\right|\left|a_{k l} b_{l k}\right| \geq\left|\operatorname{Re}\left(a_{i j} b_{j i}\right) \operatorname{Re}\left(a_{k l} b_{l k}\right)\right| .
$$

Inequality (10) was independently proven in [8]. Our proof is simpler than Kosaki's one.

As a concluding remark, we point out that [10, Theorem 1] is incorrect in general.

Remark IV.2: Reference [10, Theorem 1] is not true in general. A counterexample is given as follows. Let

$$
\rho=\frac{1}{4}\left(\begin{array}{ll}
3 & 0 \\
0 & 1
\end{array}\right), \quad A=\left(\begin{array}{cc}
0 & i \\
-i & 0
\end{array}\right), \quad B=\left(\begin{array}{ll}
0 & 1 \\
1 & 0
\end{array}\right) .
$$

Then we have, $I(\rho, A) I(\rho, B)-\left|\operatorname{Re}\left(\operatorname{Corr}_{\rho}(A, B)\right)\right|^{2}=\frac{7-4 \sqrt{3}}{4}$ and $|\operatorname{Tr}(\rho[A, B])|^{2}=1$. These imply

$$
I(\rho, A) I(\rho, B)-\left|\operatorname{Re}\left(\operatorname{Corr}_{\rho}(A, B)\right)\right|^{2}<\frac{1}{4}|\operatorname{Tr}(\rho[A, B])|^{2} .
$$

\section{ACKNOWLEDGMENT}

The authors would like to thank the reviewers for providing valuable comments to improve our manuscript.

\section{REFERENCES}

[1] J.-C. Bourin, "Some inequalities for norms on matrices and operators," Linear Alg. Appl., vol. 292, pp. 139-154, 1999.

[2] J. I. Fujii, "A trace inequality arising from quantum information theory," Linear Alg. Appl., vol. 400, pp. 141-146, 2005.

[3] P. Gibilisco and T. Isola, "On the characterization of paired monotone metrics," Ann. Inst. Stat. Math., vol. 56, pp. 369-381, 2004.

[4] _ , "On monotonicity of scalar curvature in classical and quantum information geometry," J. Math. Phys., vol. 46, pp. 1-14, 2005.

[5] M. R. Grasselli, "Duality, monotonicity and Wigner-Yanase-Dyson metrics," Inf. Dimens. Anal. Quantum Prob. Related Topics, vol. 7, pp. 215-232, 2004.

[6] W. Heisenberg, "Über den anschaulichen Inhalt der quantummechanischen Kinematik und Mechanik," Z. Phys., vol. 43, pp. 172-198, 1927.

[7] A. Jencova, "Flat connections and Wigner-Yanase-Dyson metrics," Pep. Math. Phys., vol. 52, pp. 331-351, 2003.

[8] H. Kosaki, "Matrix trace inequality related to uncertainty principle," Int. J. Math., vol. 16, pp. 629-646, 2005.

[9] E. H. Lieb, "Convex trace functions and the Wigner-Yanase-Dyson conjecture," Adv. Math., vol. 11, pp. 267-288, 1973.

[10] S. Luo and Q. Zhang, "On skew information," IEEE Trans. Inf. Theory, vol. 50, no. 8, pp. 1778-1782, Aug. 2004.

[11] _ _An informational characterization of Schrödinger's uncertainty relations," J. Statist. Phys., vol. 114, pp. 1557-1576, 2004.

[12] H. P. Robertson, "The uncertainty principle," Phys. Rev., vol. 34, pp. 163-164, 1929.

[13] E. Schrödinger, "About Heisenberg uncertainty relation," in Proc. Prussian Acad. Sci., Phys. Math., vol. XIX, 1930, p. 293. Section.

[14] E. P. Wigner and M. M. Yanase, "Information content of distribution," in Proc. Nat. Acad. Sci. U.S.A., vol. 49, 1963, pp. 910-918.

[15] — "On the positive semidefinite nature of certain matrix expression," Canad. J. Math., vol. 16, pp. 397-406, 1964. 